\title{
Études/Inuit/Studies
}

\section{Éducation et transmission des savoirs inuit au Canada}

\section{Frédéric Laugrand et Jarich Oosten}

Volume 33, numéro 1-2, 2009

Éducation et transmission des savoirs inuit au Canada

Education and transmission of Inuit knowledge in Canada

URI : https://id.erudit.org/iderudit/044957ar

DOI : https://doi.org/10.7202/044957ar

Aller au sommaire du numéro

Éditeur(s)

Association Inuksiutiit Katimajiit Inc.

Centre interuniversitaire d'études et de recherches autochtones (CIÉRA)

ISSN

0701-1008 (imprimé)

1708-5268 (numérique)

Découvrir la revue

Citer cet article

Laugrand, F. \& Oosten, J. (2009). Éducation et transmission des savoirs inuit au Canada. Études/Inuit/Studies, 33(1-2), 7-34. https://doi.org/10.7202/044957ar d'utilisation que vous pouvez consulter en ligne. 


\section{Éducation et transmission des savoirs inuit au Canada}

\section{Sous la direction de: \\ Frédéric Laugrand* et Jarich Oosten ${ }^{* *}$}

Il est temps qu'on reconnaisse à l'Esquimau ce qu'on a appelé le «droit d'être différent». Et ce droit ne sera vraiment reconnu que lorsque l'Esquimau aura son école à lui et sera instruit, dans la mesure du possible, dans sa propre langue (Mary-Rousselière 1963: 8).

Lorsque les étudiants se posent des questions sur qui ils sont présentement et où ils se dirigent, c'est grâce aux récits de vie des aînés qu'ils peuvent trouver un sens à leur culture et à eux-mêmes en tant qu’Inuit vivant dans le présent (Annahatak 1994: 17) ${ }^{1}$.

\section{Introduction}

Dans l'Arctique canadien, les toutes premières écoles font leur apparition au XIX siècle avec l'ouverture des missions chrétiennes. Les missionnaires sont convaincus que l'instruction et l'éducation religieuse facilitent la christianisation et ils espèrent vite recruter une élite capable de prendre la relève de cette première évangélisation. Dans nombre de missions anglicanes, les missionnaires forment ainsi des leaders et pasteurs autochtones, consacrant une bonne partie de leur temps à instruire les femmes et les enfants. En Terre de Baffin, par exemple, le révérend Peck et son collègue Julian Bilby font de Peter Tulugaqjuaq l'un des premiers responsables de la diffusion du christianisme. Sur place, ces membres de la Church Missionary Society enseignent le catéchisme mais aussi les mathématiques, la géographie, le dessin et la langue anglaise. Cette première scolarisation semble plutôt bien acceptée par les Inuit qui assistent aux séances avec assiduité et passent même des examens. Les missionnaires semblent également satisfaits des résultats si l'on en juge par les observations consignées dans leurs journaux et rapports. La plupart du temps, cependant, les Inuit apprennent à lire et à écrire par eux-mêmes en feuilletant les bibles qui circulent dans les camps, si bien qu'au début du $\mathrm{XX}^{\mathrm{e}}$ siècle, la population du Sud Baffin est probablement mieux instruite que celle des campagnes québécoises et canadiennes à la même époque

* CIÉRA, Université Laval, Pavillon Charles-De Koninck, local 0450, 1030 avenue des SciencesHumaines, Québec (Québec) G1V 0A6, Canada. Frederic.Laugrand@ant.ulaval.ca

** Institute of Cultural Anthropology and Development Sociology, Faculty of Social Sciences, Leiden University, Postbus 9555, 2300 RB Leyde, Pays-Bas. oosten@fsw.leidenuniv.nl

1 Toutes les traductions de l'anglais au français sont des auteurs.

ÉTUDES/INUIT/STUDIES, 2009, 33(1-2): 7-20 
(Laugrand 2002: 115-120; Laugrand et al. 2003). Dans les missions catholiques, les Oblats procèdent de façon analogue. Ils enseignent le catéchisme, la lecture et même l'écriture syllabique empruntée aux anglicans, nonobstant leurs dissensions avec ces derniers. Au Nunavut, cette forme d'éducation se maintient jusque dans les années 1950 lorsque le gouvernement, dans le cadre de sa politique de sédentarisation, décide de prendre le relais en ouvrant des écoles locales ou résidentielles.

\section{Des écoles missionnaires aux écoles résidentielles: vers l'assimilation}

Au début des années 1960, une véritable stratégie d' «assimilation par l'éducation» est mise en place. Un peu partout, des Inuit envoient leurs enfants dans des écoles sans toujours en mesurer les conséquences. Originaire de Pond Inlet, Simon Anaviapik explique avoir été intimidé et obligé de laisser partir son enfant préféré à l'école (Brody 1987: 214). De nombreuses familles font état de fortes pressions de la part des missionnaires ou des agents blancs, craignant, en cas de refus de scolariser leurs enfants, de perdre les allocations familiales qu'on leur promettait ${ }^{2}$. Pour d'autres familles, en revanche, l'école ouvrait de nouvelles possibilités.

Eu égard aux écoles résidentielles, le gouvernement fédéral a momentanément hésité sur le format à privilégier. En 1952, il avait songé à ouvrir des écoles sous la forme de grandes tentes qui pourraient accueillir des élèves une partie de l'année, soit de Pâques au début de l'été, de sorte que les enfants passent ensuite l'hiver auprès de leurs familles (King 1998: 61). Mais cette option n’a finalement pas été retenue et la décision fut prise de construire des écoles dans chaque village ou de transporter les enfants dans les toutes nouvelles écoles résidentielles.

À Chesterfield Inlet, où fut établie une des toutes premières écoles résidentielles par les Sœurs de la Charité en 1951, d'importantes discussions eurent lieu avant que le gouvernement fédéral n'en prenne la responsabilité et ne rebaptise l'institution Turquetil Hall, en 1954. Au départ, Mgr Lacroix jugeait le projet gouvernemental peu réaliste. D'abord, Chesterfield Inlet disposait déjà d'une école qui, à ses yeux, fonctionnait bien. Ensuite, il s'était déjà entendu sur un projet avec les sœurs missionnaires qu'il préférait à un personnel laïc. Enfin, il ne trouvait pas le projet du fédéral très adéquat, arguant du fait que les enfants n'apprendraient sans doute pas grand-chose en si peu de temps et qu'il était plus commode de les placer dans une école avec une résidence, que sous la tente. Contrairement au gouvernement fédéral, Mgr Lacroix souhaitait que les enfants ne fréquentent l'école résidentielle qu'entre 6-7 et 12 ans et seulement pour une période limitée, les enfants devant rentrer chez eux du $1^{\text {er }}$ janvier à Pâques (King 1998: 62). Bien que Mgr Lacroix ne pensât pas que les enfants en apprendraient plus sur la chasse, la pêche et la trappe dans leurs familles que pendant leur séjour à l'école, il était conscient, comme les missionnaires oblats, de l'importance de préserver les valeurs inuit. Aux termes de longues discussions, Mgr

2 Plusieurs témoignages présentés sur le site Internet d’Isuma à Iglulik, traduits et retranscrits par Peter Irniq, vont dans ce sens.

8/F. LAUGRAND ET J. OOSTEN 
Lacroix et le gouvernement fédéral en arrivèrent à une entente. Des professeurs seraient recrutés parmi les Sœurs de la Charité qui logeraient dans la même résidence que les élèves, arrangement qui épargnerait des dépenses au gouvernement fédéral. En échange, le fédéral obtenait que les sœurs acceptent des enfants non catholiques, que le bâtiment de l'école soit construit et entretenu aux frais du Diocèse et que les sœurs recrutées disposent des qualifications nécessaires pour y assurer un enseignement de qualité. Il était enfin convenu que ce dispositif serait réévalué en septembre 1963, date à laquelle un bilan serait établi et l'accord éventuellement reconduit. Mgr Lacroix obtenait pour sa part le maintien d'un financement du gouvernement à hauteur de $2.00 \$$ par élève et par jour afin que l'école puisse assurer le transport des enfants et leur habillement.

Aux yeux des missionnaires, l'école résidentielle de Chesterfield Inlet n’avait cependant pas pour objectif principal une assimilation totale comme le souhaitait le gouvernement fédéral. Encouragés par le cas de Sœur Pélagie, la toute première femme inuit qui venait de prononcer ses vœux, les catholiques souhaitaient surtout former une élite intellectuelle et faire naître des vocations autochtones. Dans un mot qu'il adresse «aux amis des missions esquimaudes», Mgr Lacroix justifie son projet dans les termes suivants:

Aujourd'hui, il me faut de nouveau vous tendre la main en faveur d'une oeuvre qui me tient grandement à cœur; celle de l'éducation des Esquimaux. L'heure est en effet venue pour nous de parachever le travail d'évangélisation des Esquimaux de la Baie d'Hudson en mettant à leur portée une éducation adaptée à leurs besoins en même temps que chrétienne. L'école-pensionnat est, par excellence, le moyen d'obtenir ce résultat et de former une élite esquimaude ${ }^{3}$.

Dans un autre document, Sœur Germaine Désilets se fait plus précise sur ces vocations:

Nous formulons des vœux pour que de nombreuses vocations indigènes surgissent parmi l'élite esquimaude. La fondation du pensionnat a pour but premier, nous le répétons, de former les Esquimaux au point de vue intellectuel et religieux. Mais nous espérons aussi préparer, le temps venu, des prêtres et des religieuses esquimaudes qui travailleront à la conversion de leur peuple. Cette fondation est tout à fait dans l'esprit missionnaire; établir l'Église en lui donnant des ministres de la couleur et de la langue des peuples à évangéliser ${ }^{4}$.

Ces détails sont importants à retenir. En effet, même si les projets missionnaires ont joué un rôle dans le processus d'assimilation des Inuit, leurs intentions et motivations étaient alors radicalement opposées à celles du gouvernement fédéral. Ces différences expliquent pourquoi tant de missionnaires, comme le père Guy MaryRousselière, se sont longtemps révoltés contre le projet gouvernemental de vouloir transformer les Inuit en «copies carbone» des Canadiens. Alors que l’Unesco faisait un

3 Archives Provinciales de Nicolet, Chesterfield-Pensionnat, Historique, document 35.

4 Archives Provinciales de Nicolet, Chesterfield-Pensionnat, Historique, document 40.

ÉDUCATION ET TRANSMISSION.../9 
peu partout sur la planète la promotion de l'éducation moderne et que les régions du Nord se développaient, les missionnaires se situaient donc à contre-courant, craignant déjà les effets dramatiques d’une déculturation: «Le système d’éducation actuel est orienté vers la production de ce qu'on a appelé 'l'Homo arcticus' moderne qui ne sera ni blanc ni Indien ni Esquimau [...] mais uniquement Canadien et ne parlera que l’Anglais», écrivait le père Mary-Rousselière (1963: 7-8; voir aussi Renaud 1963).

Sur le terrain, les nouvelles écoles qui sont ouvertes entre 1949 et 1962 au Québec arctique et entre 1954 et 1965 dans les Territoires du Nord-Ouest, répondent à différents modèles ${ }^{5}$. À côté des anciennes écoles missionnaires, de petits foyers sont mis en place avec une capacité de huit à 24 écoliers, les plus grands foyers étant en mesure de recevoir plus de 250 écoliers. Tous ces établissements fonctionnent soit comme des externats (c'est le cas des écoles de Baker Lake, Cape Dorset, Eskimo Point, etc.), soit comme des internats ou pensionnats qu'on retrouve dans les plus grands centres comme à Inuvik (1959) ou à Churchill (1964), mais aussi dans de petites communautés, comme à Aklavik, à Pangnirtung ou Clyde River, pour se limiter à quelques exemples. Plus ou moins fréquentées, la plupart de ces institutions fermeront leurs portes à la fin des années 1960 ou au début des années 1970, avec la mise sur pied d'un réseau d'éducation publique.

Dans toutes ces écoles locales ou résidentielles, les programmes et les curriculums sont pensés dans des perspectives étrangères aux Inuit. De 1955 aux années 1970, le Ministère du Nord canadien y importe tout simplement les programmes en vigueur dans les provinces de l'Alberta, du Manitoba et de l'Ontario. Si, avant 1958, certains programmes avaient été pensés pour favoriser l'intégration des élèves et répondre aux besoins du Nord (enseignement en anglais, apprentissage de l'écriture, développement de formations pratiques en menuiserie, en maçonnerie, etc.), la situation bascule à la fin des années 1950. Dorénavant, les spécificités du Nord ne comptent plus. Il s’agit seulement de préparer les jeunes Inuit à fonctionner dans l'économie des Qallunaat (King 1998: 114-115) et à les occidentaliser. Dans les écoles, les enseignants utilisent des textes tels que la série pour enfants Dick and Jane, entièrement construite sur des valeurs et des images de Blancs. À de rares exceptions, rien de très inuit ne figure dans les curriculums. Les jeunes élèves découvrent des images des villes et des fermes du sud, apprennent leur appartenance au vaste pays canadien et leur allégeance à sa majesté la Reine d'Angleterre Elizabeth II. Les matières principales demeurent l'anglais et l'économie domestique, cette dernière prévoyant des activités différentes pour les filles et les garçons. En somme, il faut attendre 1970, date à laquelle la gestion de l'éducation est confiée au nouveau gouvernement des Territoires du Nord-Ouest, pour voir apparaître des exercices pédagogiques avec des contenus un peu plus adaptés ${ }^{6}$.

5 Pour une étude plus complète des effectifs et des écoles résidentielles chez les Inuit, voir King (1998, 2006) et Vick-Westgate (2002).

6 En 1974, par exemple, le Department of Education publie le livre de Mark Kalluak, How Kabloonat became and other Inuit legends.

10/F. LAUGRAND ET J. OOSTEN 


\section{Vers un contrôle de l'éducation}

Au cours des années 1970 et 1980, alors que les revendications autochtones prennent plus d'ampleur, d'intenses débats sur les méthodes et les orientations à privilégier opposent missionnaires, Inuit et agents fédéraux. En 1971, la commission Hawthorn dresse un sombre tableau des politiques éducatives en milieu autochtone. Le rapport parlementaire sur l'éducation des Indiens et des Eskimo propose quelques améliorations sur la langue, mais ces mesures restent fort timides. Les missionnaires critiquent avec encore plus de véhémence la politique assimilatrice gouvernementale. À Iglulik, des initiatives voient le jour pour valoriser la transmission des savoirs locaux. Le père Mary-Rousselière écrit:

Est-il vain d'espérer que l'administration cessera de confondre éducation avec instruction et se rendra compte enfin que l'éducation ne peut être dispensée que dans un milieu favorable? Quant à la place que doivent prendre à l'école la langue et la culture esquimaude, il est encourageant de constater que devant l'inertie de la Division de l'Éducation, certains Esquimaux ont décidé de prendre en main leurs propres intérêts. C'est ainsi que la nouvelle association culturelle d’Iglulik «Inummarit» prépare déjà un programme d’éducation esquimaude et envisage la publication de certains livres de classe en esquimau. Le Ministère des Affaires du Nord a accordé une subvention à ce projet et la construction d'un vaste bâtiment à Iglulik est prévue pour l'été prochain (Mary-Rousselière 1972: 7).

Épaulé par la mission catholique locale, ce projet d'Inummarit, qui donnera lieu à plusieurs publications, est l'un des premiers du genre. Il devance à la fois des initiatives qui seront prises plus tard par l'Inuit Cultural Institute (Nunavut) et l'Institut culturel Avataq (Nunavik). Il préfigure aussi des projets culturels qui se développeront 10 ans plus tard, à l'initiative de l'Inullarit Society d'Iglulik et presque 30 ans plus tard, à l'initiative du Nunavut Arctic College, qui publiera les premiers ouvrages scolaires où des témoignages d’aînés sont présentés intégralement et utilisés par des étudiants inuit dans le cadre de leurs travaux ${ }^{7}$.

En 1975, alors que les statistiques disponibles indiquent que le nombre des enfants scolarisés est passé de 380 en 1955 à 4 375, le père Robert Lechat (1975) signe à son tour un article corrosif sur le mirage et le mythe de la scolarisation. Lechat fustige la déstructuration du tissu social dont l'école et le gouvernement qui l'appuie sont pour lui les grands responsables, mais dont les Inuit sont complices, en ne s’inquiétant pas assez du futur de leurs enfants. La pertinence des savoirs traditionnels apparaît ici encore plus évidente mais la proposition de Lechat d'envisager une éducation à géométrie variable, c'est-à-dire à plusieurs options (un curriculum axé sur ceux des écoles du sud pour ceux qui le souhaitent, un curriculum axé sur les savoirs traditionnels pour ceux qui préfèrent poursuivre leur vie de chasseurs, et un curriculum intermédiaire pour les indécis), ne sera jamais entendue. Les avis de nombreux Inuit publiés sur ce thème dans des revues comme Taqralik, Inuit Monthly ou Inuit Today ne

Voir, par exemple, les séries Interviewing Inuit Elders et Inuit Perspectives on the XXth Century lancées par Susan Sammons et Alexina Kublu dans le cadre du Nunavut Arctic College Oral Traditions, projet mis sur pied en 1996. 
le seront pas davantage, si bien que les organisations inuit réclameront encore longtemps un meilleur contrôle afin que l'éducation n'accentue pas la perte des savoirs traditionnels (voir Anonymous 1974; Ipellie 1981a, 1981b, 1982). En 1981, Alootook Ipellie faisait ainsi état de sa détermination à mieux réformer le système éducatif:

Est-ce que la culture traditionnelle des Inuit est si peu digne qu'on lui prête une attention spéciale que personne n'essaye de la préserver? [...] Il est temps de réviser le système éducationnel [...]. La réalité est que les Inuit et les Qallunaat sont des gens différents [...] ils ont des croyances et des valeurs différentes et sont élevés dans une lumière différente dès leur premier jour [...]. Les Inuit ont une tradition qui est [...] plus forte que celle des Qallunaat (Ipellie 1981a: 5-6).

Aujourd'hui, les Inuit ne jugent pas tous avec la même sévérité cette entreprise d’occidentalisation orchestrée par le biais des écoles. Les avis restent partagés et varient selon les villages, les générations et les familles. Si plusieurs critiquent vertement les écoles et regrettent les excès d'une éducation qui a coupé les jeunes de leurs traditions et des savoirs ancestraux, d'autres paraissent plus circonspects. Certains émettent même des avis très positifs, reconnaissant la qualité de l'éducation reçue, en particulier à Chesterfield Inlet ${ }^{8}$. À l'époque, certains Inuit voyaient en effet des avantages à ce que leurs enfants soient scolarisés. Plusieurs espéraient bien que ces jeunes assumeraient un jour de nouvelles responsabilités et seraient en mesure de contrôler l'exploitation des ressources locales. Dans un article publié dans la revue Imperial Oil, en octobre 1960, Abe Okpik (1960: 17) écrivait: «Aujourd’hui, pour nous, les Esquimaux, je crois que c'est l'instruction qu'il faut avant tout. Une partie des enfants sont dans de bonnes écoles, avec des instituteurs». Comme d'autres, Okpik aspirait donc à l'éducation, mais il espérait aussi que les valeurs inuit soient respectées, ce qui n’était pas vraiment le cas. À la fin des années 1980, l’idée d'une éducation mieux contrôlée par les communautés inuit est un sujet toujours âprement discuté par les premiers éducateurs inuit. David Serkoak (1989), enseignant et ancien directeur d'école à Iqaluit, aujourd'hui responsable des cours de culture au Nunavut Sivuniksavut, considérait que la responsabilité communautaire et l'implication des parents demeuraient deux points majeurs pour réussir à instaurer un système éducatif adéquat et efficace: «the success of native education is directly related to the amount of community control and involvement there is in the school system» ${ }^{9}$.

Au Nunavik, la Convention de la Baie James ouvrit de nouvelles perspectives. Dès les années 1975, les Inuit se virent confier de nouvelles responsabilités dans ce domaine. Appuyé par l'Université McGill, un programme de formation des enseignants inuit vit rapidement le jour (Cram 1985). Betsy Annahatak (1994: 16), qui en a

8 On consultera la série Life Stories of Northern Leaders pour différents témoignages sur ces expériences dans les écoles. Pour Abe Okpik, par exemple, ses trois années passées à l'école résidentielle All Saints d'Inuvik, n'ont pas été pénibles car il y a fait des rencontres et n'a pas oublié sa langue maternelle. Okpik (2005: 109-110) explique en revanche comment il a dû, à son retour dans sa communauté, réapprendre les techniques de chasse.

9 Pour une histoire et une évaluation des curriculums scolaires dans les Territoires du Nord-Ouest, voir Carney (1983, 1984); Farrow et Wilman (1989); MacPherson (1991).

12/F. LAUGRAND ET J. OOSTEN 
bénéficié, considérait que ce processus d’empowerment avait été bien accueilli parce qu'il avait permis justement une prise de contrôle de l'éducation par les Inuit euxmêmes, qui avaient décidé alors de renforcer la transmission des langues et des valeurs inuit $^{10}$.

Dans les Territoires du Nord-Ouest, l'évolution se fit plus lentement. Plusieurs enseignants firent certes assez vite preuve d'une grande capacité d'adaptation dans les écoles, prenant toutes sortes d'initiatives ${ }^{11}$. Mais il fallut en fait attendre les années 1990 et l'avènement du Nunavut pour observer une évolution importante dans les mentalités et les pratiques ${ }^{12}$. Dans les deux cas, les Inuit furent séduits par l'ambition de concilier les valeurs éducatives des Inuit avec les exigences de la société moderne en combinant les meilleurs aspects des deux systèmes, afin de bénéficier à la fois des avantages que procurent l'écriture et l'oralité, les cultures inuit et occidentales. Betsy Annahatak (1994: 17) décrit la difficulté de ce travail synthétique: «Le présent défi qui m’occupe, [...] est de développer des programmes et du matériel didactique en inuktitut pour supporter l'apprentissage, les initiatives des étudiants pour apprendre - des deux cultures - tout en maintenant des racines authentiques et en s'adaptant au monde moderne».

\section{La langue et la transmission des savoirs: comment construire un modèle plus respectueux des valeurs inuit?}

Au Nunavut, un changement de taille s'est produit en 2000, avec l'introduction progressive d'un nouveau concept, l'Inuit qaujimajatuqangit ('les savoirs toujours pertinents'), dont la préséance est depuis lors réaffirmée dans tous les documents officiels du nouveau gouvernement ${ }^{13}$. La toute récente Loi sur l'éducation du Nunavut, adoptée en 2008, inscrit elle aussi l'Inuit qaujimajatuqangit parmi ses principes fondamentaux en l'enchâssant dans son chapitre 15 à l'article 1 et en y ajoutant la liste de six autres valeurs directrices qui lui sont associées.

Aujourd'hui, les critiques des Inuit envers les méthodes d'éducation restent abondantes. Certains ne comprennent ni les méthodes employées ni les orientations pédagogiques et signalent toujours les différences qui existent entre la culture inuit et celle des Qallunnat. D’autres critiquent plutôt la faiblesse des programmes et les résultats auxquels ils conduisent, ceux-ci étant largement en dessous des standards canadiens. Inuit et non-Inuit font état de nombreux problèmes: le financement aléatoire

10 Une histoire détaillée de l'éducation au Nunavik est présentée par Forgues (1987) et surtout VickWestgate (2002). Voir Daveluy (2008) et Gauthier (1989) pour une évaluation des interventions gouvernementales en matière d'éducation au Nunavik.

11 Voir, par exemple, le récit de Joanne Tompkins (1998) qui a été la responsable d’une école à Hall Beach.

12 Plusieurs volumes sur les savoirs inuit sont produits à cette époque comme celui d'Evik-Twerdin (1991).

13 Pour une définition de ce concept, voir Therrien (2002). 
des programmes communautaires; leur manque de cohérence et d'uniformité à tous les niveaux, de leur conception à leur mise en œuvre. Le manque de soutien à l'apprentissage et le manque de formation du personnel sont d'autres problèmes qui hypothèquent la formation et favorisent le décrochage des adolescents, lequel est lui aussi largement au-dessus de la moyenne du pays. D’autres font valoir les conflits et les situations de stress que l'éducation scolaire génère dans les familles ou s'interrogent sérieusement sur la pertinence de certaines matières par rapport aux débouchés réels pour les élèves qui obtiennent leurs diplômes, sans oublier bien évidemment les craintes multiples concernant la disparition de la langue et de la culture inuit, des savoirs environnementaux et des aptitudes requises pour vivre dans l'Arctique.

Faute d'espace, il n'est guère possible d'exposer ici un bilan plus détaillé de ces gigantesques défis d'une éducation, pas plus d'ailleurs que de certaines réussites, mais de nombreux Inuit s'accordent pour dire que cette éducation n'est pas encore assez en phase avec leur culture, ni assez articulée aux exigences du monde contemporain (voir ITK 2004; 2007). Plusieurs des contributions de ce numéro d'Études/Inuit/Studies reviennent sur ces points. Rappelons seulement combien, avec la notion d'Inuit qaujimajatuqangit à laquelle tous les contributeurs de ce numéro font référence, la situation actuelle marque peut-être un nouveau tournant.

Reste à savoir comment l'objectif d' «inuitiser» les institutions, en accordant plus de place aux valeurs inuit et à l'inuktitut, pourra aboutir, les problèmes que pose son application demeurant énormes. Les dangers d'un essentialisme abusif, par exemple, sont bien présents et il serait de ce point de vue dramatique de réifier et de figer des traditions qui n’ont cessé de se modifier et d'emprunter des éléments exogènes au cours de leur histoire (Saladin d'Anglure 1988).

Dans les régions de l'Arctique canadien, l'institution scolaire a encore tout d'une institution ambivalente. Certes, des progrès importants sont observables au niveau des cycles du primaire et du secondaire, une plus grande place étant maintenant faite à la langue inuit. Au Nunavut, si les élèves reçoivent ainsi une instruction en langue inuit pendant leurs trois premières années, puis un apprentissage graduel de l'anglais les deux années suivantes, l'anglais ne devient la langue de travail qu'à partir de la $7^{\mathrm{e}}$ année. Cette valorisation de la langue et de la culture se double dans la plupart des écoles d'une plus grande place accordée aux aînés, invités à intervenir régulièrement dans les écoles et à jouer leur rôle de conseillers et détenteurs du savoir traditionnel. Pour de multiples raisons, ces interventions ne sont cependant pas faciles, comme l'indiquent encore Vera Arnatsiaq (2002), enseignante à Iglulik, et Anne-Pascale Targé (2005) dans son étude sur les écoles et la transmission des savoirs traditionnels à Arviat. Il faut ajouter le rôle d'autres institutions particulièrement dynamiques, comme le Pulaarvik Kablu Friendship Centre à Kangir\&iniq (voir Laugrand 2008) ou l'Institut culturel Avataq au Nunavik qui organisent des camps de chasse et contribuent ainsi à mieux relier les jeunes à leurs aînés. Au Nunavik, depuis la création de Kativik School Board en 1978, l'inuktitut est également enseigné aux jeunes élèves jusqu'en $2^{\mathrm{e}}$ année, ces derniers pouvant ensuite choisir le système anglais ou francophone. Mais les familles n'apportent pas toujours le meilleur appui au système scolaire où les 
problèmes de l'absentéisme et du décrochage précoce gagnent du terrain. Si l'implication des Inuit est donc indéniable dans les institutions scolaires de ces deux régions, plusieurs problèmes similaires s'y retrouvent, notamment le manque de ressources professorales et de matériaux pédagogiques adéquats.

Au secondaire et au collégial, les formations disponibles sont elles aussi plus pertinentes et plus adaptées, plus diverses aussi puisque des institutions ont même ouvert leurs portes dans le sud du Canada, notamment à Montréal (Cégep MarieVictorin, College Abbot) et à Ottawa (Nunavut Sivuniksavut). Mais bien des défis restent à relever, notamment au niveau de la transmission des valeurs et des savoirs dans un contexte qui a profondément changé et où se creuse le fossé intergénérationnel. À cet égard, le Nunavut Sivuniksavut s'est engagé dans une voie originale avec des cohortes d'étudiants qui y reçoivent à la fois un enseignement classique, ouvert sur l'étranger (comme en attestent ces voyages d'études annuels en Afrique, en Mongolie, aux îles Fidji ou au Groenland), et des cours de culture et de techniques traditionnelles.

Au-delà de tous ces changements, des défis de taille demeurent. Plusieurs formations professionnelles ne sont toujours pas accessibles aux résidents du Nord et les jeunes ayant reçu une formation complète ne sont pas assez nombreux. La plupart des postes qui exigent de grandes qualifications sont par conséquent occupés par des non-Inuit, les Inuit étant relégués à des postes moins bien rémunérés. À terme, des programmes novateurs et communautaires sont attendus dans plusieurs domaines qui vont de la santé à la justice, de l'environnement à l'industrie, sans oublier des activités de perfectionnement professionnel et de formation continue, au risque de voir apparaître rapidement de nouveaux échecs qui iront de pair avec un inévitable discrédit.

En définitive, si au primaire comme au collégial l'école est aujourd’hui mieux acceptée et valorisée pour ses mérites et ses enseignements, elle reste contestée et critiquée en raison de ses lacunes et de son interférence avec d'autres modes de transmission des savoirs. Les Inuit espèrent bien un jour obtenir des programmes plus performants et des moyens pour amener les élèves à persévérer dans leurs études, mais ils reprochent encore au système actuel de perpétuer des visées assimilatrices et d'accélérer l'érosion de l'identité culturelle inuit (ITK 2004: 3). Intitulée “There's life and then there's school": School and community as contradictory contexts for Inuit self/knowledge, la thèse d'Anne Douglas (1998) n'a pas perdu de son actualité lorsqu'elle fait ressortir les contradictions entre ces deux univers. Les Inuit espèrent toutefois y remédier d'ici 2019, date à laquelle le ministre actuel Louis Tapardjuk envisage de mettre en œuvre une éducation entièrement bilingue, construite sur les valeurs inuit et plus favorable à la transmission des savoirs. Pour le moment, les difficultés s'avèrent encore importantes sur le plan des rapports sociaux, de la conception de la vie et des formes de savoir en présence, les objectifs de l'école ne répondant pas exactement aux attentes des Inuit. Les normes sociales et les pratiques que les jeunes Inuit apprennent à l'école vont encore à l'encontre de bien des conceptions inuit qui privilégient l'observation à l'accumulation du savoir, valorisent l'autonomie de la personne et délaissent la théorie au profit d'un apprentissage plus 
empirique axé sur la pratique. Il faut admettre que ces conceptions et ce pragmatisme ne sont pas toujours bien acceptés par les non-Inuit ${ }^{14}$.

Aujourd'hui, alors que l'échec scolaire suscite de nouvelles préoccupations largement médiatisées par les journaux régionaux comme le Nunatsiaq News, qui a publié en 2002 une série d'articles sous le titre The crisis in Nunavut schools, les Inuit semblent enclins à revaloriser encore davantage leurs expériences et leurs savoirs, de même que la participation de leurs aînés. Certains instructeurs non-inuit s’inquiètent de devoir travailler et produire des matériaux pédagogiques dans sept ou huit dialectes et trois systèmes orthographiques, mais l'existence même de cette variété n'est-elle pas un signe tangible du dynamisme des traditions inuit qui, loin de disparaître sous les effets de l'éducation des années 1950, sont encore vivantes?

À une époque où l’éducation est considérée comme une priorité par Eva Aariak, la Première ministre du Nunavut, il nous semblait opportun de revenir sur ces questions. Alors que le cas des écoles résidentielles semble en effet en voie de trouver un règlement et que le gouvernement fédéral s’est excusé en juin 2008 pour cette période noire de son histoire, les Inuit, dont la population est extrêmement jeune (57\% des Inuits sont âgés de 25 ans ou moins en 2001), évoquent d'ambitieux projets. L'Inuit Language Protection Act et l'Education Act ne seraient ici que les prémisses de réformes à venir destinées à mettre en place une instruction entièrement bilingue et plus respectueuse des valeurs et des traditions inuit, les modèles provenant ici du Groenland. Mentionnons ainsi le projet de la Piqqusilirivvik Cultural School, une école qui sera ouverte dans les années à venir à Kangiqtugaapik et strictement consacrée à la transmission des savoirs traditionnels et des techniques de chasse ${ }^{15}$, ou encore ce projet d'université en voie d'émerger sous le nom de Ilitturvik University Society (Windeyer 2009).

Mais l'occasion de traiter d'éducation s'y prête d'autant plus que les discours relayés par la presse et les différents gouvernements, de même que par certaines grandes entreprises, ne sont également pas sans évoquer les argumentaires des années 1950, dans les temps forts de l'assimilation. Alors que le Nord est en effet de nouveau convoité par de multiples entreprises minières, plusieurs institutions canadiennes lorgnent sur les jeunes générations, espérant trouver là une future main-d’œuvre locale en mesure de travailler pour elles à l'exploitation de ces ressources. Nous espérons que ce numéro contribuera à cette réflexion collective sur les enjeux de l'éducation et à mieux comprendre comment elle peut jouer un rôle dans la transmission des savoirs et dans leur actualisation.

14 La présentation la plus complète de ces valeurs demeure celle de Jean Briggs (1970, 1983, 2000, 2001). Pour une présentation plus sommaire, voir Oosten et Laugrand (1999), Shaimaijuk (2000) et Petit (2003).

15 Sur des exemples d'ateliers organisés pour permettre la transmission des savoirs, voir Oosten et Laugrand (2007) et Laugrand (2008).

16/F. LAUGRAND ET J. OOSTEN 


\section{Références}

ANNAHATAK, Betsy

1994 Quality Education for Inuit today? Cultural Strengths New things, and Working out the Unknows: A story by an Inuk, Peabody Journal of Education, 69(2): 12-18.

ANONYMOUS

1974 Education: a Controversial ordinance, Inuit Monthly, 3(8): 32-36.

ARNATSIAQ, Vera

2002 Teaching Inuktitut in School: Our experiences at the Attagutaaluk Elementary School in Igloolik, Nunavut, Études/Inuit/Studies, 26(1): 181183.

BRIGGS, Jean

1970 Aspects of Inuit Value Socialization, Ottawa, Musée National du Canada.

1983 Le modèle traditionnel d'éducation chez les Inuit: différentes formes d'expérimentation face à l'inconnu, Études/Inuit/Studies, 13(1): 13-25.

2001 Qallunaat Run on Rails; Inuit Do What They Want To Do. Autonomies in Camp and Town, Études/Inuit/Studies, 25(1-2): 13-25.

BRIGGS, Jean (dir.)

2000 Childrearing Practices, Iqaluit, Nunavut Arctic College, Interviewing Inuit Elders, 3.

BRODY, Hugh

1987 Living Arctic: Hunters of the Canadian North, Vancouver, Douglas and McIntyre.

CARNEY, Robert J.

1983 Inuit Rights and Inuit School Programs: A Study in Political Socialization. New Education, 5(2): 61-62.

1984 Northwest Territories teacher education program evaluation, Yellowknife, GNWT Education.

CRAM, Jack

1985 Northern Teachers for Northern Schools: An Inuit Teacher-Training Program, McGill Journal of Education, 20(2): 113-131. 
DAVELUY, Michelle

2008 Le français et la scolarisation des Inuit du Nunavik (Canada), in P. Dalley, et S. Roy (dir.), Francophonie, minorités et pédagogie, Ottawa, Presses de l’Université d’Ottawa: 75-100.

DOUGLAS, Anne S.

1998 "There's life and then there's school": School and community as contradictory contexts for Inuit self/knowledge, thèse de doctorat, McGill University, Montréal.

EVIK-TWERDIN, Leena

1991 Traditional Inuit Beliefs in Stories and Legends, Iqaluit, Nortext/Baffin Divisional Board of Education.

FARROW, Malcom et D. WILMAN

1989 Self Determination in Native Education in the Circumpolar North, Yellowknife, GNWT.

FORGUES, Louis

1987 L'action du gouvernement fédéral chez les Inuit du Nouveau-Québec: le cas de l'éducation (1949-1975), mémoire de maîtrise, Ste-Foy, Université Laval.

GAUTHIER, Bernard

1989 Évaluation des interventions gouvernementales en matière d'éducation au Nouveau-Québec inuit, Recherches amérindiennes au Québec, 19(1): 63-80.

IPELLIE, Alootook

1981a Northern Education. One year Sabbatical Needed, Inuit Today, 9: 4-6.

1981b Inuit Legends as teaching Tools, Inuit Today, 9: 40-43.

1982 Education in the North, Inuit Today, 10, 1: 2-17.

INUIT TAPIRIIT KANATAMI (ITK)

2004 Document d'information sur les Inuits et l'éducation, Document de travail pour les séances de suivi sectoriel sur l'apprentissage continu, les 13 et 14 novembre à Winnipeg et les 18 et 19 novembre à Ottawa, Ottawa, Inuit Tapiriit Kanatami.

2007 Inuit Education in Canada, in Indigenous Standards in Education, Ottawa, Inuit Tapiriit Kanatami: 9-13.

KALLUAK, Mark

1974 How Kabloonat became and other Inuit legends, Yellowknife, Department of Education.

18/F. LAUGRAND ET J. OOSTEN 
KING, David

1998 The History of the Federal Residential Schools for Inuit, located in Chesterfield Inlet, Yellowknife, Inukvik and Churchill, 1955-1970, thèse de doctorat, Trent University, Peterborough.

2006 A Brief Report of the Federal Government of Canada's Residential School System for Inuit, report prepared for the Aboriginal Healing Foundation, Ottawa, Aboriginal Healing Foundation.

LAUGRAND, Frédéric, Jarich OOSTEN et Maaki KAKKIK

2003 Keeping the Faith, Iqaluit, Nunavut Arctic College/Nortext, Memory and History in Nunavut, 3.

LAUGRAND, Frédéric

2002 Mourir et renaître. La réception du christianisme par les Inuit de l'Arctique de l'Est canadien, Québec, Presses de l’Université Laval, Leiden, CNWS.

2008 L'école de la Toundra. Réflexions sur l'éducation à partir de quelques ateliers de transmission des savoirs avec des aînés et des jeunes inuit, Cahiers du CIÉRA, 1: 77-96.

LECHAT, Robert

1975 The Mirage of Schooling, Eskimo, 9: 5-9.

MacPHERSON, Norman John

1991 Dreams and Visions: Education in the Northwest Territories From Early Days to 1984, Yellownknife, Department of Education, Government of the Northwest Territories.

MARY-ROUSSELIÈRE, Guy

1963 Where Do We Stand With Eskimo Education?, Eskimo, 66: 5-12.

1972 Le rapport parlementaire sur l'éducation indienne et esquimaude et quelques commentaires, Eskimo, 3: 3-7.

OKPIK, Abraham

2005 We Call It Survival, Louis McComber (dir.), Iqaluit, Nunavut Arctic College, Life Stories of Northern Leaders, 1.

OOSTEN, Jarich et Frédéric LAUGRAND

2007 Surviving in Different Worlds: Transferring Inuit Traditions from Elders to Youth, Iqaluit, Nunavut Arctic College. 
PETIT, Céline

2003 L'éducation et la scolarisation au Nunavik et au Nunavut: transmission et réappropriation des savoirs, in $\mathrm{P}$. Huret (dir.), Les Inuit de l'Arctique Canadien, Québec, CIDEF-AFI/Inukshuk: 207-226.

RENAUD, André

1963 Éducation des Esquimaux, Eskimo, 66: 3-4.

SALADIN D’ANGLURE, Bernard

1988 Les Inuit à l'école de Rabelais, de Descartes ou de Lévi-Strauss? Regard anthropologique sur l'éducation interculturelle, in F. Ouellet (dir.), Pluralisme et école. Jalons pour une approche critique de la formation interculturelle des éducateurs, Québec, Institut de la recherche sur la culture: 436-464.

SERKOAK, David

1989 Community Control in Native Education. A Sense of Ownership, Responsibility and Commitment, in M. Farrow et D. Wilman, Self Determination in Native Education in the Circumpolar North, Yellowknife, GNWT.

SHAIMAIJUK, Julia

2000 L'instruction des enfants, in J. Oosten et F. Laugrand (dir.), Introduction, Iqaluit, Nunavut Arctic College, Entrevues avec des aînés, 1: 154-157.

TARGÉ, Anne-Pascale

2005 Transmission des savoirs oraux dans les écoles inuit: étude du cas de la communauté d'Arviat, mémoire de maîtrise, Québec, Université Laval.

THERRIEN, Michèle

2002 La question du concept d'Inuit qaujimajatuqangit et le bilan du jeune gouvernement du Nunavut, Études canadiennes / Canadian Studies, 52: 131-147.

TOMPKINS, Joanne

1998 Teaching in a Cold and Windy place: Change in an Inuit School, Toronto, University of Toronto Press.

VICK-WESTGATE, Ann

2002 Nunavik: Inuit controlled education in Arctic Quebec, Calgary, University of Calgary Press.

WINDEYER, Chris

2009 New group pushes for Iqaluit-based university, Nunatsiaq News, July 28. 


\title{
Education and transmission of Inuit knowledge in Canada
}

\section{Guest editors: \\ Frédéric Laugrand ${ }^{*}$ and Jarich Oosten ${ }^{* *}$}

\begin{abstract}
It is time that the Eskimos be recognized as having what is called "the right to be different." And this right will truly be recognised only when the Eskimos have their own schools and are educated, as far as possible, in their own language (Mary-Rousselière 1963: 8). ${ }^{1}$

Once students face the questions of who they are now and where they are going, it is within the real life stories of elders that they can make meaning of our culture and of themselves as Inuit living in the present (Annahatak 1994: 17).
\end{abstract}

\section{Introduction}

In Arctic Canada, the very first schools appeared in the 19th century with the opening of the Christian missions. The missionaries were convinced that a religious education and upbringing would make Christianisation easier and hoped for speedy recruitment of an elite that could take over this first wave of evangelisation. At many Anglican missions, the missionaries trained Aboriginal leaders and pastors, devoting much of their time to educating women and children. On Baffin Island, for example, Reverend Peck and his colleague Julian Bilby made Peter Tulugaqjuaq one of the very first ones in charge of spreading Christianity. These members of the Church Missionary Society locally taught catechism and also mathematics, geography, drawing, and English. This initial attempt at schooling seemed to be rather well accepted by the Inuit, who assiduously attended the sessions and even some exams. The missionaries likewise seemed satisfied with the results, judging by observations in their journals and reports. Usually, however, the Inuit learned to read and write on their own by leafing through Bibles that circulated in the camps. In the early 20th century, the South Baffin population was probably better educated than were rural Quebeckers and Canadians of the same period (Laugrand 2002: 115-120; Laugrand et al. 2003). At the Catholic missions, the Oblates proceeded similarly. They taught catechism, reading, and even syllabic writing, which they had borrowed from the Anglicans notwithstanding the

* CIÉRA, Université Laval, Pavillon Charles-De Koninck, local 0450, 1030 avenue des SciencesHumaines, Québec, Québec G1V 0A6, Canada. Frederic.Laugrand@ant.ulaval.ca

** Institute of Cultural Anthropology and Development Sociology, Faculty of Social Sciences, Leiden University, Postbus 9555, 2300 RB Leiden, The Netherlands. oosten@fsw.leidenuniv.nl

1 This text and all its French citations were translated into English by Peter Frost.

ÉTUDES/INUIT/STUDIES, 2009, 33(1-2): 21-34 
dissensions between the two churches. In Nunavut, this form of education continued until the 1950s, when the government, as part of its sedentarisation policy, decided to move into this field by opening local or residential schools.

\section{From missionary schools to residential schools: toward assimilation}

In the early 1960s, a real strategy of "assimilation through education” was adopted. Almost everywhere, Inuit sent their children to schools without always knowing the consequences. A native of Pond Inlet, Simon Anaviapik says he was intimidated and forced to let his favourite child go to school (Brody 1987: 214). Many families recall being pressured by White missionaries or agents. If they refused to have their children educated, they feared losing the family allowances they had been promised. ${ }^{2}$ For other families, however, school opened up new opportunities.

With regard to residential schools, the federal government hesitated awhile on the form they should take. In 1952, it had thought about opening schools in big tents that students could attend part of the year, from Easter to early summer. They could then spend the winter with their families (King 1998: 61). But this option was ultimately rejected in favour of building schools in each village or transporting the children to recently built residential schools.

At Chesterfield Inlet, where the Sisters of Charity had established one of the first residential schools in 1951, there was much discussion before the federal government took over and renamed it Turquetil Hall in 1954. Mgr. Lacroix initially judged the government project to be unrealistic. First, Chesterfield Inlet already had a school that worked well in his opinion. Second, he had already agreed to a project with the missionary nuns, whom he preferred to a secular teaching staff. Finally, he did not find the federal project very appropriate, arguing that the children would probably not learn much in so little time and would benefit more from a school with a residence than from one in a tent. Unlike the federal government, Mgr. Lacroix wished to see the children attend residential school only from 6-7 to 12 years of age and only over a limited school year. They would thus stay home from January 1 to Easter (King 1998: 62). Although Mgr. Lacroix did not believe that the children would learn more about hunting, fishing, and trapping in their families than during their stay at school, he was aware, like the Oblate missionaries, of the importance of preserving Inuit values. Following long discussions, Mgr. Lacroix and the federal government came to an agreement. Teachers would be recruited from the Sisters of Charity and live in the same residence as the students, thus saving the federal government money. In exchange, the federal government obtained several concessions: the nuns would accept non-Catholic children, the school would be built and maintained at the expense of the Diocese, and the teaching nuns would have the qualifications needed to provide a quality education. It was finally agreed that this arrangement would be reassessed in September 1963,

2 This view is supported by several accounts on the website of Isuma (based in Iglulik), which Peter Irniq has translated and retranscribed.

22/F. LAUGRAND AND J. OOSTEN 
when a review would be carried out and the agreement possibly extended. For his part, Mgr. Lacroix obtained that government funding would be kept at \$2.00 per student per day so that the school could provide the children with transportation and clothing.

In the missionaries' eyes, the Chesterfield Inlet residential school did not have total assimilation as its main goal, as the federal government wished. Encouraged by the case of Sister Pélagie, the very first Inuit nun, who had just taken her vows, the Catholics especially hoped to train an intellectual elite while bringing about Aboriginal vocations. In a message to "the friends of the Eskimo missions," Mgr. Lacroix justified his project in the following terms:

Today, I must again extend my hand to you for an activity that I hold dear to my heart: Eskimo education. The time has come for us to finish off the work of evangelising the Eskimo of Hudson Bay by putting within their reach an education that meets their needs and is at the same time Christian. The boarding school is, par excellence, the way to obtain this result and to train an Eskimo elite. ${ }^{3}$

In another document, Sister Germaine Désilets more precisely outlined these vocations:

We are formulating vows for the emergence of many Aboriginal vocations among the Eskimo elite. The founding of the boarding school has the primary goal, we repeat, of training Eskimos from an intellectual and religious standpoint. But we also hope to prepare, when the time comes, Eskimo priests and nuns who will work at converting their people. This founding is fully in the missionary spirit; establishing the Church by giving it ministers of the colour and language of the peoples to be evangelised. ${ }^{4}$

These details are worth keeping in mind. Even though the missionaries' projects played a role in the process of assimilating the Inuit, their intentions and motivations were at that time radically at odds with those of the federal government. These differences explain why so many missionaries, like Father Guy Mary-Rousselière, were long opposed to the government's project of turning the Inuit into "carbon copy" Canadians. At a time when UNESCO was promoting modern education almost all around the world and the northern regions were being developed, the missionaries were bucking the trend. They already feared the dramatic effects of deculturation: "The current educational system is geared to producing what is called modern 'Homo arcticus' who will be neither White nor Indian nor Eskimo [...] but uniquely Canadian and will speak only English,” wrote Father Mary-Rousselière (1963: 7-8; see also Renaud 1963).

The new schools opened between 1949 and 1962 in Arctic Quebec, and between 1954 and 1965 in the Northwest Territories. They followed different models. ${ }^{5}$

3 Archives Provinciales de Nicolet, Chesterfield-pensionnat, Historique, document 35.

4 Archives Provinciales de Nicolet, Chesterfield-Pensionnat, Historique, document 40.

5 For a more complete study of Inuit residential schools and their students, see King (1998, 2006) and Vick-Westgate (2002). 
Alongside the old missionary schools, small schoolhouses were erected with a capacity of eight to 24 students, the largest ones being able to hold over 250. All of them were run either as day schools (as with the ones at Baker Lake, Cape Dorset, Eskimo Point, etc.) or as residential or boarding schools in larger centres like Inuvik (1959) and Churchill (1964) or smaller communities like Aklavik, Pangnirtung, and Clyde River to name just a few. After having varying levels of attendance, most of them closed in the late 1960 s or early 1970 s with the creation of a public educational system.

In all of the local or residential schools, the programs and curricula reflected outlooks that were foreign to the Inuit. From 1955 to the 1970s, Canada's Department of Northern Affairs simply imported programs used in the provinces of Alberta, Manitoba, and Ontario. Although before 1958 some programs had been designed to encourage student integration and to meet Northern needs (teaching in English, learning of writing, development of practical training in woodworking, masonry, etc.), the situation reversed in the late 1950s. From then on, the distinctiveness of the North no longer mattered. The aim was simply to prepare Inuit youth for life in a Qallunaat economy (King 1998: 114-15) and to Westernise them. In the schools, the teachers used books like the children's series Dick and Jane, which was entirely constructed on White values and images. With few exceptions, nothing very Inuit appeared in the curricula. Young students discovered pictures of southern towns and farms, learned that they belonged to the vast country of Canada and owed allegiance to her majesty the Queen of England Elizabeth II. The main subjects remained English and home economics, which assigned girls and boys gendered activities. Not until 1970, when the new Northwest Territories government was given management of education, did classroom exercises begin to appear with content a bit more adapted to the local reality. ${ }^{6}$

\section{Toward control of education}

During the 1970s and 1980s, with the rise of Aboriginal activism, intense debate over methods and aims pitted missionaries, Inuit, and federal agents against each other. In 1971, the Hawthorn Commission painted a dark picture of Aboriginal education policies. The parliamentary report on Indian and Eskimo education proposed several improvements to language instruction, but these measures remained quite timid. The missionaries criticised the government's policy of assimilation even more vehemently. In Iglulik, initiatives were begun to promote the transmission of local knowledge. Mary-Rousselière wrote:

Is it vain to hope that the administration will stop confusing education with instruction and finally realise that education can be provided only in favourable surroundings? As for the place of Eskimo language and culture at school, it is encouraging to see that when faced with the inertia of the Division of Education, some Eskimos have decided to take charge of their own interests. Thus, Iglulik's new cultural association "Inummarit" is already

6 In 1974, for example, the Department of Education published a book by Mark Kalluak, How Kabloonat became and other Inuit legends.

24/F. LAUGRAND AND J. OOSTEN 
preparing an Eskimo education program and is planning to publish some classroom books in Eskimo. The Department of Northern Affairs has granted funding for this project and the construction of a large building in Iglulik is scheduled for next summer (Mary-Rousselière 1972: 7).

Backed by the local Catholic mission, Inummarit's project would give rise to several publications and was one of the very first projects of this kind. It ushered in later initiatives by the Inuit Cultural Institute (Nunavut) and the Avataq Cultural Institute (Nunavik). It also heralded cultural projects that would develop 10 years later, under the leadership of the Inullarit Society of Iglulik and almost 30 years later under the auspices of Nunavut Arctic College, which would publish the first schoolbooks in which accounts by elders are presented in full and used by Inuit students in their class work. ${ }^{7}$

In 1975, when available statistics indicated that the number of children in school had risen from 380 in 1955 to 4,375, Father Robert Lechat (1975) in turn wrote a caustic article on the mirage and myth of schooling. He denounced the destructuring of the social fabric, which he mainly attributed to the schools and the government that supported them. The Inuit too were parties to this process by not being concerned enough about the future of their children. He particularly stressed the relevance of traditional knowledge, but his words fell on deaf ears when he called for a versatile educational system, i.e., one with several options (a curriculum based on southern school curricula for those who so wished, a curriculum based on traditional knowledge for those who preferred to continue living as hunters, and an intermediate curriculum for the undecided). The opinions of many Inuit on this subject, published in magazines like Taqralik, Inuit Monthly, or Inuit Today, likewise went unheeded, so much so, that long afterward Inuit organisations were still calling for better control to keep education from accelerating the loss of traditional knowledge (see Anonymous 1974; Ipellie 1981a, 1981b, 1982). In 1981, Alootook Ipellie (1981a: 5-6) affirmed his determination to improve the educational system:

Is the traditional culture of the Inuit so unworthy of special attention that no one is attempting to preserve it? [...] It is time to overhaul the educational system [...]. The reality is that Inuit and Qallunaat are different people [...] they have different beliefs and values and are brought up in a different light from day one [...]. Inuit have a tradition that is [...] stronger than the Qallunaat's.

Today, not all Inuit are so severe in judging this orchestrated effort to Westernise via the schools. Opinions are mixed and vary by village, by generation, and by family. Although many harshly criticise the schools and regret the excesses of an education that cut young people off from their traditions and ancestral knowledge, others appear to be more circumspect. Some even give very positive opinions, acknowledging the quality

7 See, for example, the series Interviewing Inuit elders and Inuit perspectives on the XXth Century launched by Susan Sammons and Alexina Kublu for the Nunavut Arctic College Oral Traditions Project started in 1996. 
of the education they received, in particular at Chesterfield Inlet. ${ }^{8}$ At the time, some Inuit saw benefits in their children receiving an education. Many hoped these young people would one day take on new responsibilities and be able to control the development of local resources. In an Imperial Oil magazine article from October 1960, Abe Okpik (1960: 17) wrote: “Today, for us, the Eskimo, I think education is what is needed above all. Part of the children are in good schools, with teachers." Like others, Okpik yearned for education, but also hoped that Inuit values would be respected. This did not really happen. In the late 1980s, the idea of education being better controlled by Inuit communities was a subject still bitterly discussed by the first Inuit educators. David Serkoak (1989), a teacher and former school principal at Iqaluit, today in charge of culture courses at Nunavut Sivuniksavut, felt that community responsibility and parental involvement remained two major points for success in creating an adequate and effective educational system: "the success of native education is directly related to the amount of community control and involvement there is in the school system."'9

In Nunavik, the James Bay and Northern Quebec Agreement opened up new prospects. As early as 1975, the Inuit were being given new responsibilities in this field. With the support of McGill University, an Inuit teacher-training program was soon set up (Cram 1985). According to one graduate, Betsy Annahatak (1994: 16), this empowerment process was greeted favourably because it enabled the Inuit to take control of their education. They then decided to do more to pass down Inuit languages and values to the next generation. ${ }^{10}$

In the Northwest Territories, change was slower. Many teachers certainly acted soon enough to adapt their classroom teaching through all sorts of initiatives. ${ }^{11}$ But not until the 1990s, and the advent of Nunavut, were there major changes in mentalities and practices. ${ }^{12}$ The Inuit were seduced by the ambition of reconciling Inuit educational values with the requirements of modern society. They wished to combine the best of both: oral traditions and Inuit culture with written traditions and Western culture. Betsy Annahatak (1994: 17) described the problems involved in combining the two: "The present challenge which I am involved in, [...] is to develop programs and instructional materials in Inuktitut that will support the learning, and initiatives of students to learn,

8 See the series Life Stories of Northern Leaders for different accounts about these experiences in the schools. For Abe Okpik, for example, his three years at All Saints residential school in Inuvik were not distressing. He made friends and acquaintances there and did not forget his mother tongue. On the other hand, Okpik (2005: 109-110) explains how he had to relearn hunting techniques after returning to his community.

9 For a history and assessment of school curricula in the Northwest Territories, see Carney (1983, 1984); Farrow and Wilman (1989); MacPherson (1991).

10 A detailed history of education in Nunavik is presented by Forgues (1987) and especially VickWestgate (2002). See Daveluy (2008) and Gauthier (1989) for an assessment of government actions on education in Nunavik.

11 See, for example, the account by Joanne Tompkins (1998) who ran a school at Hall Beach.

12 Several volumes on Inuit knowledge were produced at that time, such as one by Evik-Twerdin (1991).

26/F. LAUGRAND AND J. OOSTEN 
from both cultures - maintaining an authentic root as well as adapting some of the modern ways."

\section{Language and transmission of knowledge: How to build a model that better respects Inuit values?}

In Nunavut, a major change came in 2000 with the progressive introduction of a new concept, Inuit qaujimajatuqangit ('knowledge that is still relevant'), whose primacy has since been reaffirmed in all official documents of the new government. ${ }^{13}$ The recent Education Act of Nunavut, passed in 2008, likewise entrenches Inuit qaujimajatuqangit among its fundamental principles in Chapter 15, Article 1 and adds it to a list of six other associated guiding values.

Today, the Inuit are still highly critical of educational methods. Some understand neither the methods nor the aims and still point to the differences that exist between Inuit culture and Qallunaat culture. Others instead criticise the weakness of the programs and the results they lead to, these being well below Canadian standards. Inuit and non-Inuit report many problems: hit-and-miss funding of community programs and the lack of consistency and uniformity at all levels, from design to implementation. Inadequate learning support and staff training are other problems that incur educational costs later on and that encourage teenagers to drop out, this too being well above the Canadian mean. Others point to conflicts and stressful situations that schooling generates in families or seriously wonder about the relevance of some subjects to actual job opportunities for graduating students, not to mention of course the many fears over the extinction of Inuit language and culture, environmental knowledge, and skills needed for living in the Arctic.

For lack of space, it is scarcely possible to list these huge educational challenges in more detail, no more than some of the successes. Many Inuit agree, however, that this education is still not fully in tune with their culture, nor is it sufficiently connected to the requirements of the present-day world (see ITK 2004; 2007). These points are raised in several contributions to this issue of Études/Inuit/Studies. Suffice it to say that the notion of Inuit qaujimajatuqangit, which appears in all of the contributions, may mark a new turning point in the current situation.

It remains to be seen how the goal of "Inuitising” the schools may be reached through greater emphasis on Inuit values and Inuktitut. The implementation problems remain huge. For example, there are the dangers of abusive essentialism. It would be tragic to reify these traditions and freeze them for all time, since they have always been changing and have continually borrowed exogenous elements throughout their history (Saladin d'Anglure 1988).

13 For a definition of this concept, see Therrien (2002). 
In the regions of Arctic Canada, schools still play an ambivalent role. Much progress is certainly observable in the elementary and secondary grades, there being much more emphasis now on the Inuit language. In Nunavut, students are taught in the Inuit language for the first three grades and then increasingly in English over the next two. English is the language of instruction only from Grade 7 onward. Emphasis on language and culture is combined in most schools with a greater place for elders, who are regularly invited to schools and act as advisors and bearers of traditional knowledge. For many reasons, this role is not easy, as indicated by Vera Arnatsiaq (2002), an Iglulik teacher, and by Anne-Pascale Targé (2005) in her study of schools and the transmission of traditional knowledge at Arviat. We should also mention other especially dynamic institutions, like the Pulaarvik Kablu Friendship Centre at Kangir\&iniq (see Laugrand 2008) and Avataq Cultural Institute in Nunavik, which organise hunting camps and thus help better connect young people to their elders. In Nunavik, since creation of the Kativik School Board in 1978, Inuktitut has also been taught to young students up to Grade 2. They can then choose the English or French system. The families, however, do not always provide the school system with the best support, and problems with absenteeism and early dropping out are gaining ground. Although the Inuit are undeniably involved in the educational institutions of both regions, several problems are also common to both, notably lack of teaching staff and lack of adequate teaching materials.

At the high school and junior college levels, the available programs are more relevant, better adapted, and more diverse too. Some are now even available in southern Canada, notably in Montreal (Cégep Marie-Victorin, Abbot College) and in Ottawa (Nunavut Sivuniksavut). But many challenges remain, particularly when it comes to transmitting values and knowledge in a context that has seen profound change and a widening of the generation gap. In this respect, Nunavut Sivuniksavut has taken an original approach of providing student cohorts with an education that is both conventional and open to the world (as seen in annual study tours to Africa, Mongolia, Fiji, or Greenland) while offering courses in culture and traditional technologies.

Beyond all of these changes, there are still major challenges. Several programs for learning a trade are not always available to northern residents and not enough young people are fully educated. Most highly qualified positions are consequently filled by non-Inuit, the Inuit being relegated to positions that pay less. Eventually, novel community programs are expected in several fields from health care to justice and from the environment to industry, not to mention retraining and continuing education activities. There is, however, the risk of new failures that will inevitably discredit the programs.

Clearly, although education is now better accepted and valued for its benefits and content at the elementary and junior college levels, it is still being questioned and criticised for its shortcomings and its interference with other means of passing down knowledge. The Inuit hope one day to have improved programs and ways to help children persevere in their education, but they still reproach the current system for perpetuating goals of assimilation and for accelerating the erosion of Inuit cultural 
identity (ITK 2004: 3). With its title “There's life and then there's school”: School and community as contradictory contexts for Inuit self/knowledge, the dissertation by Anne Douglas (1998) still holds true today when it points out the contradictions between these two worlds. The Inuit nonetheless hope to remedy this situation by 2019, when the current minister Louis Tapardjuk plans to implement fully bilingual education that will reflect Inuit values and be more conducive to passing down knowledge. For now, there are still serious problems in terms of current social relations, outlook on life, and forms of knowledge. Educational goals do not exactly match Inuit expectations. The social norms and practices that young Inuit learn at school still clash with many Inuit ones, i.e., valuing observation over accumulating knowledge, emphasising personal autonomy, and attaching less importance to theory than to an empirical, practice-based approach to learning. Admittedly, such values and pragmatism are poorly accepted by non-Inuit. ${ }^{14}$

Today, academic failure is fuelling new concerns in regional papers like Nunatsiaq News, which in 2002 ran a series of articles under the title The crisis in Nunavut schools. In response, the Inuit seem inclined to look to their own experiences and knowledge, and to participation by their elders. Some non-Inuit teachers worry about having to work with and produce teaching materials in seven or eight dialects and three spelling systems. But is not the very existence of this variety a tangible sign of the dynamism of Inuit traditions, which, far from being wiped out by education in the 1950s, are still alive?

At a time when Eva Aariak, the Premier of Nunavut, has made education a priority, it seems to us opportune to come back to these questions. The case of residential schools seems on the way to being settled and the federal government apologised in June 2008 for this dark period of its history. Meanwhile, ambitious projects are being put forward by the Inuit, whose population is extremely young (57\% of all Inuit were 25 years old or younger in 2001). The Inuit Language Protection Act and the Education Act are merely a basis for further reforms that will lead to an entirely bilingual education that better respects Inuit values and traditions, the models being brought over from Greenland. There is also the Piqqusilirivvik Cultural School, which will open some years from now in Kangiqtugaapik and be strictly devoted to the transmission of traditional knowledge and hunting techniques. ${ }^{15}$ Finally, there is a project to create a university under the name of the Ilitturvik University Society (Windeyer 2009).

The time is ripe to discuss education, all the more so because the press, the different governments, and some major businesses are talking about it in a way that harks back to what was being said in the 1950s, at the height of pressures to assimilate. At a time when many mining companies are again coveting the North, some of

14 These values are most completely presented by Jean Briggs (1970, 1983, 2000, 2001). For a more summary presentation, see Oosten and Laugrand (1999), Shaimaijuk (2000), and Petit (2003).

15 For examples of workshops on the transmission of knowledge, see Oosten and Laugrand (2007) and Laugrand (2008). 
Canada's institutions are eyeing the younger generations in the hope of having a future local workforce that can assist in developing these resources. We hope this issue of Inuit Studies will help people think collectively about the challenges of education and better understand its role in the transmission of knowledge and bringing it up to date.

\section{References}

ANNAHATAK, Betsy

1994 Quality Education for Inuit today? Cultural Strengths, New things, and Working out the Unknowns: A story by an Inuk, Peabody Journal of Education, 69(2): 12-18.

ANONYMOUS

1974 Education: a Controversial ordinance, Inuit Monthly, 3(8): 32-36.

ARNATSIAQ, Vera

2002 Teaching Inuktitut in School: Our experiences at the Attagutaaluk Elementary School in Igloolik, Nunavut, Études/Inuit/Studies, 26(1): 181183.

BRANT, C.S. and C.W. HOBART

1966 Eskimo Education, Danish and Canadian: A Comparison, Canadian Review of Sociology / Revue canadienne de sociologie, 3(2): 47-66.

BRIGGS, Jean

1970 Aspects of Inuit Value Socialization, Ottawa, National Museums of Canada.

1983 Le modèle traditionnel d'éducation chez les Inuit: différentes formes d'expérimentation face à l'inconnu, Études/Inuit/Studies, 13(1): 13-25.

2001 Qallunaat Run on Rails; Inuit Do What They Want To Do. Autonomies in Camp and Town, Études/Inuit/Studies, 25(1-2): 13-25.

BRIGGS, Jean (ed.)

2000 Childrearing Practices, Iqaluit, Nunavut Arctic College, Interviewing Inuit Elders, 3.

BRODY, Hugh

1987 Living Arctic: Hunters of the Canadian North, Vancouver, Douglas and McIntyre.

CARNEY, Robert J.

1983 Inuit Rights and Inuit School Programs: A Study in Political Socialization. New Education, 5(2): 61-62. 
CARNEY, Robert J.

1984 Northwest Territories teacher education program evaluation, Yellowknife, GNWT Education.

CRAM, Jack

1985 Northern Teachers for Northern Schools: An Inuit Teacher-Training Program, McGill Journal of Education, 20(2): 113-131.

DAVELUY, Michelle

2008, Le français et la scolarisation des Inuit du Nunavik (Canada), in P. Dalley, and S. Roy (ed.), Francophonie, minorités et pédagogie, Ottawa, Presses de l’Université d'Ottawa: 75-100.

DOUGLAS, Anne S.

1998 "There's life and then there's school": School and community as contradictory contexts for Inuit self/knowledge, $\mathrm{PhD}$ dissertation, McGill University, Montreal.

EVIK-TWERDIN, Leena

1991 Traditional Inuit Beliefs in Stories and Legends, Iqaluit, Nortext/Baffin Divisional Board of Education.

FARROW, Malcom and D. WILMAN

1989 Self-Determination in Native Education in the Circumpolar North, Yellowknife, GNWT.

FORGUES, Louis

1987 L'action du gouvernement fédéral chez les Inuit du Nouveau-Québec: le cas de l'éducation (1949-1975), master's thesis, Ste-Foy, Université Laval.

GAUTHIER, Bernard

1989 Évaluation des interventions gouvernementales en matière d'éducation au Nouveau-Québec inuit, Recherches amérindiennes au Québec, 19(1): 63-80.

IPELLIE, Alootook

1981a Northern Education. One year Sabbatical Needed, Inuit Today, 9: 4-6.

1981b Inuit Legends as teaching Tools, Inuit Today, 9: 40-43.

1982 Education in the North, Inuit Today, 10, 1: 2-17.

INUIT TAPIRIIT KANATAMI

2004 Background paper on the Inuit and education, working paper for sectorbased sessions on continuing education, November 13 and 14, Winnipeg, and November 18 and 19, Ottawa. October 20, 2004, Ottawa, Inuit Tapiriit Kanatami. 
2007 Inuit Education in Canada, in Indigenous Standards in Education, Ottawa, Inuit Tapiriit Kanatami: 9-13.

KALLUAK, Mark

1974 How Kabloonat became and other Inuit legends, Yellowknife, Department of Education.

KING, David

1998 The History of the Federal Residential Schools for Inuit, located in Chesterfield Inlet, Yellowknife, Inukvik and Churchill, 1955-1970, PhD dissertation, Trent University, Peterborough.

2006 A Brief Report of the Federal Government of Canada's Residential School System for Inuit, report prepared for the Aboriginal Healing Foundation, Ottawa, Aboriginal Healing Foundation.

LAUGRAND, Frédéric, Jarich OOSTEN and Maaki KAKKIK

2003 Keeping the Faith, Iqaluit, Nunavut Arctic College/Nortext, Memory and History in Nunavut, 3.

LAUGRAND, Frédéric

2002 Mourir et renaître. La réception du christianisme par les Inuit de l'Arctique de l'Est canadien, Québec, Presses de l’Université Laval, Leiden, CNWS.

2008 L'école de la Toundra. Réflexions sur l'éducation à partir de quelques ateliers de transmission des savoirs avec des aînés et des jeunes inuit, Cahiers du CIÉRA, 1: 77-96.

LECHAT, Robert

1975 The Mirage of Schooling, Eskimo, 9: 5-9.

MacPHERSON, Norman John

1991 Dreams and Visions: Education in the Northwest Territories From Early Days to 1984, Yellowknife, Department of Education, Government of the Northwest Territories.

MARY-ROUSSELIÈRE, Guy

1963 Where Do We Stand With Eskimo Education?, Eskimo, 66: 5-12.

1971 A New Element in Eskimo Education, Eskimo, 1: 4-6.

1972 Le rapport parlementaire sur l'éducation indienne et esquimaude et quelques commentaires, Eskimo, 3: 3-7. 
OKPIK, Abraham

2005 We Call It Survival, Louis McComber (ed.), Iqaluit, Nunavut Arctic College, Life Stories of Northern Leaders, 1.

OOSTEN, Jarich and Frédéric LAUGRAND

2007 Surviving in Different Worlds: Transfering Inuit Traditions from Elders to Youth, Iqaluit, Nunavut Arctic College.

PETIT, Céline

2003 L'éducation et la scolarisation au Nunavik et au Nunavut: transmission et réappropriation des savoirs, in P. Huret (ed.), Les Inuit de l'Arctique Canadien, Québec, CIDEF-AFI/Inukshuk: 207-226.

RENAUD, André

1963 Éducation des Esquimaux, Eskimo, 66: 3-4.

SALADIN D’ANGLURE, Bernard

1988 Les Inuit à l'école de Rabelais, de Descartes ou de Lévi-Strauss? Regard anthropologique sur l'éducation interculturelle, in F. Ouellet (ed.), Pluralisme et école. Jalons pour une approche critique de la formation interculturelle des éducateurs, Québec, Institut de la recherche sur la culture: 436-464.

SERKOAK, David

1989 Community Control in Native Education. A Sense of Ownership, Responsibility and Commitment, in M. Farrow and D. Wilman, Self Determination in native Education in the Circumpolar North, Yellowknife, GNWT.

SHAIMAIJUK, Julia

2000 L'instruction des enfants, in J. Oosten and F. Laugrand (eds), Introduction, Iqaluit, Nunavut Arctic College, Entrevues avec des aînés, 1: 154-157.

TARGÉ, Anne-Pascale

2005 Transmission des savoirs oraux dans les écoles inuit: étude du cas de la communauté d'Arviat, master's thesis, Université Laval, Québec.

THERRIEN, Michèle

2002 La question du concept d'Inuit qaujimajatuqangit et le bilan du jeune gouvernement du Nunavut, Études canadiennes/Canadian Studies, 52: 131147.

TOMPKINS, Joanne

1998 Teaching in a Cold and Windy place: Change in an Inuit School, Toronto, University of Toronto Press. 
VICK-WESTGATE, Ann

2002 Nunavik: Inuit controlled education in Arctic Quebec, Calgary, University of Calgary Press.

WINDEYER, Chris

2009 New group pushes for Iqaluit-based university, Nunatsiaq News, July 28. 